\title{
Star-cluster formation and evolution
}

\author{
Pavel Kroupa \\ Argelander Institute for Astronomy, University of Bonn, Auf dem Hügel 71, D-53347 Bonn, \\ Germany \\ email: pavel@astro.uni-bonn.de
}

\begin{abstract}
Star clusters are observed to form in a highly compact state and with low starformation efficiencies, and only 10 per cent of all clusters appear to survive to middle- and old-dynamical age. If the residual gas is expelled on a dynamical time the clusters disrupt. Massive clusters may then feed a hot kinematical stellar component into their host-galaxy's field population thereby thickening galactic disks, a process that theories of galaxy formation and evolution need to accommodate. If the gas-evacuation time-scale depends on cluster mass, then a power-law embedded-cluster mass function may transform within a few dozen Myr to a mass function with a turnover near $10^{5} M_{\odot}$, thereby possibly explaining this universal empirical feature. Discordant empirical evidence on the mass function of star clusters leads to the insight that the physical processes shaping early cluster evolution remain an issue of cutting-edge research.
\end{abstract}

Keywords. methods: n-body simulations; stars: luminosity function, mass function; stars: formation; binaries: general; globular clusters: general; open clusters and associations: general; Galaxy: structure; Galaxy: evolution; galaxies: star clusters

\section{Early cluster evolution}

The star-formation efficiency (sfe), $\epsilon \equiv M_{\text {ecl }} /\left(M_{\text {ecl }}+M_{\text {gas }}\right)$, where $M_{\text {ecl }}, M_{\text {gas }}$ are the mass in freshly formed stars and residual gas, respectively, is $0.2 \lesssim \epsilon \lesssim 0.4$ (Lada \& Lada (2003)) implying that the physics dominating the star-formation process on scales $<10 \mathrm{pc}$ is stellar feedback. Within this volume, the pre-cluster cloud core contracts under self gravity thereby forming stars ever more vigorously, until feedback energy suffices to halt the process (feedback-termination, Weidner \& Kroupa (2006)). This occurs on one to a few crossing times $\left(\approx 10^{6} \mathrm{yr}\right)$, and since each proto-star needs about $10^{5} \mathrm{yr}$ to accumulate about 95 per cent of its mass (Wuchterl \& Tscharnuter (2003)), the assumption may be made that the embedded cluster is mostly virialised at feedback-termination. Its stellar velocity dispersion,

$$
\sigma \approx \sqrt{G M_{\mathrm{ecl}} /(\epsilon R)}
$$

may then reach $\sigma=40 \mathrm{pc} / \mathrm{Myr}$ if $M_{\mathrm{ecl}}=10^{5.5} M_{\odot}$ which is the case for $\epsilon R<1 \mathrm{pc}$. This is easily achieved since the radius of one-Myr old clusters is $R \approx 1 \mathrm{pc}$ with a weak, if any dependence on mass (Bastian et al. (2005)). Very young clusters (age $\lesssim 10 \mathrm{Myr}$ ) would thus appear super-virial, i.e. with a velocity dispersion too large for the cluster mass.

The above exercise demonstrates that the possibility may be given that a hot kinematical component could add to a galactic disk as a result of clustered star formation for reasonable physical parameters. Thickened galactic disks may result. But this depends on

(i) $\epsilon$,

(ii) $R$ (cluster concentration) and

(iii) the ratio of the gas-expulsion time-scale to the dynamical time of the embedded cluster, $\tau_{\text {gas }} / t_{\text {cross }}$. 


\subsection{Empirical constraints}

The first (i) of these is clearly fulfilled: $\epsilon<40$ per cent (Lada \& Lada (2003)). The second (ii) also appears to be fulfilled such that clusters with ages $\lesssim 1$ Myr have $R \lesssim 1$ pc independently of their mass. Some well-studied cases are tabulated and discussed in Kroupa (2005). Finally, the ratio $\tau_{\text {gas }} / t_{\text {cross }}$ (iii) remains uncertain but critical.

The well-observed cases discussed in Kroupa (2005) do indicate that the removal of most of the residual gas does occur within a cluster-dynamical time, $\tau_{\text {gas }} / t_{\text {cross }} \lesssim 1$. Examples noted are the Orion Nebula Cluster (ONC) and R136 in the LMC both having significant super-virial velocity dispersions. Other examples are the Treasure-Chest cluster and the very young star-bursting clusters in the massively-interacting Antennae galaxy which appear to have HII regions expanding at velocities such that the cluster volume may be evacuated within a cluster dynamical time.

A simple calculation of the amount of energy deposited by an $\mathrm{O}$ star within a cluster crossing time into its surrounding cluster-nebula also suggests it to be larger than the nebula binding energy (Kroupa (2005)). Furthermore, Bastian \& Goodwin (2006) note that many young clusters have a radial-density profile signature as expected if they are expanding rapidly.

Thus, the data suggest the ratio $\tau_{\text {gas }} / t_{\text {cross }}$ to be near one, but much more observational work needs to be done to constrain this number. Measuring the kinematics in very young clusters would be an extremely important undertaking, because the implications of $\tau_{\text {gas }} / t_{\text {cross }} \lesssim 1$ are dramatic.

To demonstrate these implications it is now assumed that a cluster is born in a very compact state $(R \approx 1 \mathrm{pc})$, with a low sfe $(\epsilon<0.4)$ and $\tau_{\text {gas }} / t_{\text {cross }} \lesssim 1$. As noted in Kroupa (2005), "in the presence of $\mathrm{O}$ stars, explosive gas expulsion may drive early cluster evolution independently of cluster mass".

\section{Implications}

\subsection{Cluster evolution and the thickening of galactic disks}

As one of the important implications, a cluster in the age range of $\approx 1-50 \mathrm{Myr}$ will have an unphysical $M / L$ ratio because it is out of dynamical equilibrium rather than having an abnormal stellar IMF (Bastian \& Goodwin (2006)).

Another implication would be that a Pleiades-like open cluster would have been born in a very dense ONC-type configuration and that, as it evolves, a "moving-group-I" is established during the first few dozen Myr comprising roughly 2/3rds of the initial stellar population and expanding outwards with a velocity dispersion which is a function of the pre-gas-expulsion configuration (Kroupa, Aarseth \& Hurley (2001)). These computations were in fact the first to demonstrate, using high-precision $N$-body modelling, that the redistribution of energy within the cluster during the embedded phase and the expansion phase leads to the formation of a substantial remnant cluster despite the inclusion of all physical effects that are disadvantageous for this to happen (explosive gas expulsion, Galactic tidal field and mass loss from stellar evolution).

Thus, in this scenario stars form in very compact clusters that have radii less than about $1 \mathrm{pc}$ and masses larger than a dozen $M_{\odot}$, and in the presence of $\mathrm{O}$ stars the residual gas is removed explosively leading to loss of stars from the cluster which form a moving group I (an expanding population of sibling stars). A cluster re-forms after gas expulsion as a result of energy-equipartition during the embedded phase, or during the expanding phase, and fills its Roche lobe in the Galactic tidal field. Such a cluster appears with an expanded core radius (compare the $\mathrm{ONC}$ with about $0.2 \mathrm{pc}$ and the Pleiades with $1.3 \mathrm{pc}$ ) and evolves 
secularly through evaporation until it's demise (Baumgardt \& Makino (2003), Lamers et al. (2005a), Lamers et al. (2005b)). A "moving-group-II" establishes during this stage as the "classical" moving group made-up of stars which slowly diffuse/evaporate out of the re-virialised cluster remnant with relative kinetic energy close to zero. Under unfavorable conditions no cluster re-forms after gas expulsion. Such conditions may arise from timevariable tidal fields (e.g. through nearby cloud formation) or simply through unfavorable density profiles just before gas-expulsion (Boily \& Kroupa (2003a), Boily \& Kroupa $(2003 b))$, and they may dominate over the favorable ones $(\S 2.3)$. Note that the number of stars in moving-group-I always outnumber the number of stars in moving-group-II, while the unfavourable conditions would lead to no moving-group-II.

Moving-groups-I would be populated by stars that carry the initial kinematical state of the birth configuration into the field of a galaxy. Each generation of star clusters would, according to this picture, produce overlapping moving-groups-I (and II), and the overall velocity dispersion of the new field population can be estimated by adding in quadrature all expanding populations. This involves an integral over the embeddedcluster mass function, $\xi_{\mathrm{ecl}}\left(M_{\mathrm{ecl}}\right)$, which describes the distribution of the stellar mass content of clusters when they are born (Kroupa (2002), Kroupa (2005)). It is known to be a power-law (Lada \& Lada (2003), Hunter et al. (2003), Zhang \& Fall (1999)). The integral can be calculated for a first estimate of the effect. The result is that for reasonable upper cluster mass limits in the integral, $M_{\text {ecl }} \lesssim 10^{5} M_{\odot}$, the observed agevelocity dispersion relation of Galactic field stars in the solar neighbourhood can be reproduced.

This theory can thus explain the "energy deficit", namely that the observed kinematical heating of field stars with age cannot, until now, be explained by the diffusion of orbits in the Galactic disk as a result of scattering on molecular clouds, spiral arms and the bar (Jenkins (1992)). Because the age-velocity-dispersion relation for Galactic field stars increases with stellar age, this notion can also be used to map the star-formation history of the Milky-Way disk by resorting to the observed correlation between the star-formation rate in a galaxy and the maximum star-cluster mass born in the population of young clusters (Weidner, Kroupa \& Larsen (2004)).

A possible cosmologically-relevant implication of this "popping-cluster" model emerges as follows (Kroupa (2002), Kroupa (2005)): A thin galactic disk which experiences a significant star burst such that star clusters with masses ranging up to $10^{6} M_{\odot}$ can form in the disk, will puff-up and obtain a thick-disk made-up of the fast-moving velocity wings of the expanding clusters after residual gas expulsion. A notable example where this process may have lead to thick-disk formation is the galaxy UGC 1281 (Mould (2005)), which, by virtue of its LSB character, could not have obtained its thick disk as a result of a merger with another galaxy. Elmegreen \& Meloy Elmegreen (2006) observe edge-on disk galaxies with a chain-like morphology in the HST ultra-deep field finding many to have thick disks. These appear to be derived from kpc-sized clumps of stars. These clumps are probably massive star-cluster complexes which form in perturbed or intrinsically unstable gas-rich and young galactic disks. The individual clusters in such complexes would "pop" as in the theory above (Fellhauer \& Kroupa (2005)), leading naturally to thick disks as a result of vigorous early star formation.

\subsection{Structuring the initial cluster mass function}

Another potentially important implication from this theory of the evolution of young clusters is that if the gas-expulsion time-scale and/or the sfe varies with initial (embedded) cluster mass, then an initially featureless power-law mass function of embedded clusters 
will rapidly evolve to one with peaks, dips and turnovers at "initial" cluster masses that characterize changes in the broad physics involved, such as the gas-evacuation time-scale.

Note that the "embedded" cluster mass is the birth mass in stellar content, while the "initial" cluster mass is the mass of stars the virialised post-gas-expulsion star cluster would have had if it had been born with a SFE of 100 per cent $(\epsilon=1)$.

"Initial" cluster masses are derived by dynamically evolving observed clusters backwards in time to $t=0$ by taking into account stellar evolution and a galactic tidal field but not the birth process ("classical" evolution tracks, eg. Portegies Zwart et al. (2001), Baumgardt \& Makino (2003)).

To further quantify this issue, Kroupa \& Boily (2002) assumed that the function

$$
M_{\mathrm{icl}}=f_{\mathrm{st}} M_{\mathrm{ecl}},
$$

exists, where $M_{\mathrm{ecl}}$ is as above, $M_{\mathrm{icl}}$ is the "classical initial cluster mass" and

$$
f_{\mathrm{st}}=f_{\mathrm{st}}\left(M_{\mathrm{ecl}}\right) \text {. }
$$

Thus, for example, for the Pleiades, $M_{\mathrm{cl}} \approx 1000 M_{\odot}$ at the present time (age: about $100 \mathrm{Myr}$ ), and a classical initial model would place the initial cluster mass at $M_{\mathrm{icl}} \approx$ $1500 M_{\odot}$ by using standard $N$-body calculations to quantify the secular evaporation of stars from an initially bound and virialised "classical" cluster (Portegies Zwart et al. (2001)). If, however, the sfe was 33 per cent and the gas-expulsion time-scale was comparable or shorter than the cluster dynamical time, then the Pleiades would have been born in a compact configuration resembling the ONC and with a mass of embedded stars of $M_{\mathrm{ecl}} \approx 4000 M_{\odot}($ Kroupa, Aarseth \& Hurley $(2001))$. Thus, $f_{\mathrm{st}}\left(4000 M_{\odot}\right)=0.38$ in this particular case.

By postulating that there exist three basic types of embedded clusters, namely

- clusters without $\mathrm{O}$ stars (type I: $M_{\text {ecl }} \lesssim 10^{2.5} M_{\odot}$, e.g. Taurus-Auriga pre-main sequence stellar groups, $\rho \mathrm{Oph})$,

- clusters with a few O stars (type II: $10^{2.5} \lesssim M_{\text {ecl }} / M_{\odot} \lesssim 10^{5.5}$, e.g. the ONC), and

- clusters with many $\mathrm{O}$ stars and with a velocity dispersion comparable to the sound velocity of ionized gas (type III: $M_{\text {ecl }} \gtrsim 10^{5.5} M_{\odot}$ ),

it can be argued that $f_{\text {st }} \approx 0.5$ for type I, $f_{\text {st }}<0.5$ for type II and $f_{\text {st }} \approx 0.5$ for type III.

The reason for the high $f_{\text {st }}$ values for types I and III is that gas expulsion from these clusters may be longer than the cluster dynamical time because there is no sufficient ionizing radiation for type I clusters, or the potential well is too deep for the ionized gas to leave (type III clusters). Type I clusters are excavated through the cumulative effects of stellar radiation and outflows, while type II clusters probably require multiple supernovae of type II to unbind the gas (Goodwin (1997)). According to the present notion, type II clusters undergo a disruptive evolution and witness a high "infant mortality rate" (Lada \& Lada (2003)), therewith being the pre-cursors of OB associations and open Galactic clusters.

Under these conditions and an assumed inverted Gaussian functional form for $f_{\text {st }}=$ $f_{\text {st }}\left(M_{\text {ecl }}\right)$, the power-law embedded cluster mass function transforms into a cluster mass function with a turnover near $10^{5} M_{\odot}$ and a sharp peak near $10^{3} M_{\odot}$ (Kroupa \& Boily (2002)). This form is strongly reminiscent of the initial globular cluster mass function which is inferred by e.g. Vesperini (1998), Vesperini (2001), Parmentier \& Gilmore (2005), Baumgardt \& Makino (2003) to be required for a match with the evolved cluster mass function that is seen to have a universal turnover near $10^{5} M_{\odot}$. Thus, $\approx 10^{10} \mathrm{yr}$-old cluster populations have log-normal Gaussian mass functions quite independently of the environment. 
In the theory presented here, the power-law embedded cluster mass function evolves in the first $10^{7-8} \mathrm{yr}$ to the "initial" cluster mass function with the broad turn-over near $10^{5} M_{\odot}$. In this context it is of interest to note that de Grijs et al. (2003) found a turnover in the cluster mass function near $10^{5} M_{\odot}$ for clusters with an age near $10^{9} \mathrm{yr}$ in the starburst galaxy M82. This result was challenged, but in their section 2.1 de Grijs et al. (2005) counter the criticisms. This age of 1 Gyr is too young to be explainable through secular evolution starting from a power-law cluster mass function. In contrast to this interpretation, the theory outlined here would allow a power-law embedded cluster mass function to evolve to the observed form within 1 Gyr. Evaporation through two-body relaxation in a time-variable tidal field then takes over thereby enhancing the observed log-normal shape of the old cluster mass function (e.g. Vesperini (1998), Vesperini (2001), Parmentier \& Gilmore (2005)).

This ansatz may thus bear the solution to the long-standing problem that the initial cluster mass function needs to have this turnover, while the observed mass functions of very young clusters are featureless power-law distributions.

\subsection{Cluster disruption independently of mass}

If, on the other hand, $f_{\text {st }}=$ constant such that only about 10 per cent of all clusters survive independently of mass, then the above long-standing problem would remain unsolved. This situation is suggested by the results of Lada \& Lada (2003) and Fall et al. (2005), among others. Observations show that 90 per cent of all very young clusters dissolve within about $10^{4} \mathrm{yr}$, independently of their mass. This may either be due to a constant $f_{\text {st }}$, or, as pointed out by Fall et al. (2005), a result of 90 per cent of all clusters dissolving and only the rest surviving intact. The former case would simply imply a constant shift of the power-law mass function along the mass axis to smaller masses as a result of cluster disruption through stellar feedback (operating in all embedded clusters), while the latter would imply a purely stochastic element to the disruption of clusters.

It would therefore appear that this 'high infant mortality of clusters independently of cluster mass' scenario would be in contradiction with the results of de Grijs et al. (2005) on the M82 1 Gyr-old cluster system combined with the observed power-law mass function of very young clusters.

\section{Conclusions}

Observations show that star clusters of any mass are formed extremely compact $(\lesssim 1 \mathrm{pc})$, while older clusters appear well distended with radii of a few-to-many pc. Very young clusters seem to be super-virial, and expanding HII shells also indicate the explosive removal of residual gas. Star-cluster formation thus appears to be rather violent leading to a loss of a large fraction of stars and probably in many cases total cluster disruption.

As this discussion shows, the formation process of star clusters may have significant cosmological implications in that the morphology of galaxies may be shaped by the clusters born within them: the disks of galaxies are probably thickened during periods of high star-formation rates while low-mass dwarf galaxies may attain halos of stars as a result of the violent processes associated with cluster birth as a result of which stars may spread outwards with relatively high velocity dispersions. Particular examples of such events have been noted above. If this is true can be verified by measuring the velocity dispersions of stars in clusters younger than at most a few Myr. This is very important to do because thickened galactic disks are often taken to be evidence for the merging of cold-dark-matter sub-structures. Naturally, in-falling dwarf galaxies will lead to thickened disks, and counter-rotating thick disks would be prime examples of such 
processes (Yoachim \& Dalcanton (2005)), but this discourse shows that sub-pc-scale processes would probably need to be incorporated in any galaxy-evolution study.

The mass function of old globular clusters has a near-universal turnover near $10^{5} M_{\odot}$ which has so far defied an explanation, given that the mass function of very young clusters is observed to be a power-law, and that the modelling of classical disruption through two-body relaxation and tidal fields poses a challenge for evolving a power-law to the approximate log-normal form for old clusters. Again, mass-dependent processes related to the physics of residual gas expulsion can explain such a turnover, but in this case the turnover would have to become evident in cluster populations younger than about 100 Myr. The results of de Grijs et al. (2005) suggest this to be the case. The results of Zhang \& Fall (1999), however, appear to show no such evidence for the cluster population in the Antennae galaxies: the mass function for two age groups of clusters (younger than about $10 \mathrm{Myr}$, and between 10 and $160 \mathrm{Myr}$ ) show the same power-law form, albeit with different normalisations as a result of the high infant mortality rate. If this is true, then orbital anisotropies in the young globular cluster populations as proposed by Fall \& Zhang (2001) would be needed to evolve a power-law mass function to the log-normal form.

It therefore appears that the broad principles of early cluster evolution remain quiet unclear. Given that clusters are the fundamental building blocks of galaxies (Kroupa (2005)) this is no satisfying state of affairs. On the positive side, the importance of star clusters to stellar populations and other cosmological issues (merging histories of galaxies, galactic morphology) means that much more theoretical and observational effort is needed to clarify which processes act during the early life of a star cluster, and how these affect its appearance at different times.

\section{Acknowledgements}

I thank the Jan Palouš and Bruce Elmegreen for organising a great meeting in Prague.

\section{References}

Bastian, N. \& Goodwin, S. P. 2006, MNRAS 369, L9

Bastian, N., Gieles, M., Lamers, H. J. G. L. M., Scheepmaker, R. A. \& de Grijs, R. 2005, A\&A 431,905

Baumgardt, H. \& Makino, J. 2003, MNRAS 340, 227

Boily, C. M. \& Kroupa, P. 2003a, MNRAS 338, 665

Boily, C. M. \& Kroupa, P. 2003b, MNRAS 338, 673

de Grijs, R., Bastian, N. \& Lamers, H. J. G. L. M. 2003, ApJL 583, L17

de Grijs, R., Parmentier, G. \& Lamers, H. J. G. L. M. 2005, MNRAS 364, 1054

Fall, S. M. \& Zhang, Q. 2001, ApJ 561, 751

Elmegreen, B. G. \& Meloy Elmegreen, D. 2006, ApJ 650, 644

Fall, S. M., Chandar, R. \& Whitmore, B. C. 2005, ApJL 631, L133

Fellhauer, M. \& Kroupa, P. 2005, ApJ 630, 879

Goodwin, S. P. 1997, MNRAS 284, 785

Hunter, D. A., Elmegreen, B. G., Dupuy, T. J. \& Mortonson, M. 2003, AJ 126, 1836

Jenkins, A. 1992, MNRAS 257, 620

Kroupa, P. 2002, MNRAS 330, 707

Kroupa, P. 2005, in: C. Turon, K. S. O'Flaherty \& M. A. C. Perryman (eds.), The ThreeDimensional Universe with Gaia (ESA SP-576), 629

Kroupa, P. \& Boily, C. M. 2002, MNRAS 336, 1188

Kroupa, P., Aarseth, S. J. \& Hurley, J. 2001, MNRAS 321, 699

Lada, C. J. \& Lada, E. A. 2003, ARA\& $A$ 41, 57

Lamers, H. J. G. L. M., Gieles, M. \& Portegies Zwart, S. F. 2005a, A\&\&A 429, 173 
Lamers, H. J. G. L. M., Gieles, M., Bastian, N., Baumgardt, H., Kharchenko, N. V. \& Portegies Zwart, S. 2005b, A\&SA 441, 117

Mould, J. 2005, AJ 129, 698

Parmentier, G. \& Gilmore, G. 2005, MNRAS 363, 326

Portegies Zwart, S. F., McMillan, S. L. W., Hut, P. \& Makino, J. 2001, MNRAS 321, 199

Vesperini, E. 1998, MNRAS 299, 1019

Vesperini, E. 2001, MNRAS 322, 247

Weidner, C. \& Kroupa, P. 2006, MNRAS 365, 1333

Weidner, C., Kroupa, P. \& Larsen, S. S. 2004, MNRAS 350, 1503

Wuchterl, G. \& Tscharnuter, W. M. 2003, A\& A 398, 1081

Yoachim, P. \& Dalcanton, J. J. 2005, ApJ 624, 701

Zhang, Q. \& Fall, S. M. 1999, ApJL 527, L81

\section{Discussion}

ELMEGREEN: I think your idea that small massive clusters pop to form a thick disk needs some refinement. It is usually true that things which form by gravitational instabilities and collapse from the ambient ISM have velocity dispersions less than the ambient value. The dispersions also get smaller with smaller size. What we see at high redshift is that kpc-size massive clusters dissolve into a thick disk.

WALBORn: The velocity dispersion of R136 in 30 Dor may be much less than $50 \mathrm{~km} / \mathrm{s}$; it has not been determined observationally because of the difficulties of measuring small radial velocities in OB spectra with few broad lines, contaminated by nebular emission and a high fraction of spectroscopic binaries (If that number derived from the nebular emission lines, that is driven by stellar winds and is irrelevant to the stellar velocity dispersion.)

KRoupA: The argument is very simple: before cluster formation the ambient ISM velocity dispersion is $\sim 10 \mathrm{~km} \mathrm{~s}^{-1}$. But once $\sim 10^{6} \mathrm{M} \odot$ are assembled within $\sim 1 \mathrm{pc}$, then the dispersion is greater than $10 \mathrm{~km} \mathrm{~s}^{-1}$ (virial theorem). If residual gas can be removed on a crossing time sale, then a substantial part of this dispersion may be carried into the field (thickened disks). Concerning R136, I agree that the velocity dispersion of say A or $\mathrm{F}$ stars needs to be measured to check the high value obtained for O stars.

Goldman: How important is energy dissipation either by dynamical friction or turbulencehydro drag, in phases I and II?

KROUPA: It ought to be considered because the stars are moving through a dense gaseous medium, but I have not looked at this problem in much detail as it is very complex. A useful reference here would be Just, Kegel, \& Weiss (1986, A\&A).

MiEske: Have you made a quantitative calculation with respect to your suggestion that the turnover of the globular cluster luminosity function is caused by the dependence of initial mass loss on mass?

KroupA: We computed simple models in Kroupa \& Boily (2002), and detailed modeling is in progress.

ZINNECKER: You mentioned mass segregation only briefly. Would you care to comment which effect you expect on cluster evolution for initial versus dynamical mass segregation? Which one (initial or dynamical) do you favor? 
KROUPA: I personally favor primordial mass segregation because it is intuitive. However, to show this is really true we need to test the hypothesis that it is not. Dynamical mass segregation contracts the core concentrating the massive stars thus maximizing the effects of stellar evolution (cluster expansion). At the same time the cluster expands (to balance the energy of the contracting core) allowing a larger fraction of stars to become unbound compared to the case of primordial mass segregation. However, the violent and disruptive effects of expulsion of residual gas probably mostly dominates the energetics of earlier cluster evolution. 